\title{
Encontros Bibli: Revista Eletrônica de Biblioteconomia e Ciência da Informação: análisis y evolución
}

\author{
Edna Lúcia da Silva \\ Liliane Vieira Pinheiro \\ Estera Muszkat Menezes \\ Universidade Federal de Santa Catarina (Brasil)
}

\section{Resumen}

Esta investigación tuvo como meta buscar fuentes para una mejor comprensión de la producción del conocimiento en el área de la ciencia de la información en Brasil, a través del análisis de parte de esta producción difundida a través de Encontros Bibli: Revista Eletrônica de Biblioteconomia e Ciência da Informação. Este estudio analiza su estructura, sus autores, los artículos publicados, su evolución y su desarrollo como vehículo de difusión de la producción de conocimiento. La investigación ha verificado las características de esta revista electrónica como canal de difusión del conocimiento producido en la ciencia de la información en Brasil.

Palabras clave: Revista científica. Ciencia de la información. Biblioteconomía. Producción del conocimiento. Brasil.

\section{Abstract}

This research had the objective to understand the production of knowledge in information science in Brazil through the analysis of part of its scientific production disseminated through the journal Encontros Bibli: Revista Eletrônica de Biblioteconomia e Ciência da Informação. This work verified the characteristics of this electronic journal as a channel of dissemination of scientific production in information and library science in Brazil. Its structure, authors, articles, and its evolution and development as a vehicle of dissemination of knowledge are analyzed.

Keywords: Scientific journal. Information science. Library science. Production of knowledge. Brasil.

\section{Introducción}

La madurez de un campo científico puede ser evaluada de muchas maneras. Una de las más comunes es a través de la gran cantidad de programas de posgrado

Scire. 12 : 2 (jul.-dic. 2006) 99-117. ISSN 1135-3716. 
reconocidos en el área para la formación de investigadores y de canales activos de diseminación de la producción del conocimiento.

En Brasil, actualmente, la ciencia de la información pertenece al área de ciencias sociales aplicadas en la clasificación del Consejo Nacional de Desarrollo Científico y Tecnológico (CNPq). En esta área existen siete programas de posgrado autorizados por el Ministerio de la Educación del país, a través de la Coordinación de Perfeccionamiento de Personal de Nivel Superior (CAPES), en las siguientes instituciones: Pontifícia Universidade Católica de Campinas - SP (maestría), Universidade Federal da Bahia (maestría), Universidade Federal de Santa Catarina (maestría), Universidade Estadual de São Paulo de Marília - SP (maestría), Universidade de Brasília - DF (maestría y doctorado), Universidade Federal de Minas Gerais (maestría y doctorado) y Universidade Federal Fluminense/Instituto Brasileiro de Informação em Ciencia e Tecnologia - RJ (maestría y doctorado). Las publicaciones consolidadas para la diseminación de la producción científica del área existentes en el país son Ciência da Informação, Perspectivas em Ciência da Informação, Informação \& Sociedade, DataGramaZero, Transinformação, Informação \& Informação, Revista Digital de Biblioteconomia e Ciência da Informação y Encontros Bibli.

Los canales de difusión de la producción son esenciales para la actividad científica, pues desempeñan un papel crucial en la divulgación de los resultados de las investigaciones. La revista científica (o periódico) es el medio de comunicación formal más utilizado y reconocido por la comunidad científica. Investigadores clásicos de la sociología de la ciencia ya reconocieron el valor de las publicaciones periódicas. Price (1974) afirma que la revista científica es el principal medio para registrar el conocimiento humano. Ziman (1979) argumenta que se ha convertido en el principal evento de la historia de la ciencia moderna como mecanismo de publicación sistemática de fragmentos de trabajo científico.

La Associação Brasileira de Normas Técnicas (2003, p. 3) define una publicación periódica como aquella "editada en fascículos con designación numérica y/o cronológica, en intervalos prefijados (periodicidad), por tiempo indeterminado, con la colaboración, en general, de diversas personas, en la que se tratan asuntos diversos dentro de una política editorial definida".

Herschman (1970), un autor igualmente clásico del área, reconoce tres funciones de las publicaciones periódicas: la función memoria, la función diseminación y la función social. La primera se les confiere cuando representan el instrumento de registro oficial y público de la ciencia; la segunda, cuando se constituyen en instrumento de difusión de informaciones; y la tercera, cuando en cuanto institución social otorga prestigio y recompensa a los autores, miembros de redacción y editores. 
Müeller (1999) atribuye cuatro funciones básicas a la revista científica: “certificar" el conocimiento mediante el aval de la comunidad científica, permitir la comunicación entre los científicos y la divulgación más amplia de la ciencia, servir de archivo o de memoria científica y registrar la autoría del descubrimiento científico.

Garcia y Targino (1999) consideran la revista como un elemento esencial para la comunidad científica, pues preserva y difunde rápidamente el conocimiento y establece la prioridad científica.

La importancia de la revista como medio de comunicación científica ya es una opinión consolidada entre los académicos. Los autores son unánimes cuando afirman que la revista es fundamental en la actividad científica para la divulgación de resultados de investigación, y es garantía de prioridad y de reconocimiento público de los autores; estas funciones se han mantenido a lo largo del tiempo. Muchos autores también se han referido a Internet como una alternativa viable para la comunicación científica. Meadows (1999, p. 36) constata que en el desarrollo de la revista científica impresa fue necesario crear mecanismos que ofreciesen soluciones a algunos problemas, como por ejemplo la necesidad de presentar la información de una manera unificada, el mantenimiento de mecanismos de control de calidad, el establecimiento de prioridades, la distribución de un gran número de ejemplares en el ámbito internacional, etcétera. Una publicación electrónica, según este autor, puede adoptar estrategias similares a las ya adoptadas por las publicaciones impresas y, al ser un medio más flexible, puede solucionar problemas relativos a los costos de producción y a la lentitud del proceso editorial. Las publicaciones científicas electrónicas en Internet, para Sabbatini (1999, p. 1), “constituyen actualmente uno de los temas de mayor repercusión dentro de la comunicación científica, y por consiguiente de la propia ciencia moderna, cuando actúan dentro de un campo clave para el funcionamiento del actual modelo de producción del conocimiento científico, el sistema de publicaciones científicas". Oliveira (1996) argumenta que, aunque se reconociera la importancia y la función de las publicaciones periódicas en la ciencia, se hizo necesario sustituir la forma tradicional de la revista por otras formas alternativas, como la edición electrónica. Las publicaciones impresas, parte esencial de la estructura de la comunicación científica, están resultando afectadas por los medios electrónicos, y la revista científica no constituye una excepción. En la actualidad, el medio electrónico surge como una herramienta útil y ágil de intercambio de información científica, ya que posibilita el contacto entre investigadores distribuidos por todo el globo.

Las ventajas de las publicaciones periódicas electrónicas han sido analizadas en la literatura nacional por diferentes autores. Oliveira (1996) y Moutinho, Cunha Filho y Lima (2002) les atribuyen como ventaja la velocidad de difusión y los bajos costos de producción y distribución. Dias (2002, p. 21) coincide con los anteriores y añade que "el medio electrónico ofrece una flexibilidad difícil de reproducir 
hasta el momento en el medio impreso, sea por la posibilidad de hallar de forma casi instantánea cualquier palabra aislada presente en un artículo de periódico, sea por el uso de las expresiones booleanas". Para Targino (2000), el proceso electrónico de almacenamiento y recuperación, aun sin tener la rigidez del medio impreso, no es tan frágil como el de las charlas o las presentaciones orales, pues posibilita la impresión, lo que garantiza la conservación de la información para su utilización posterior. Sabbatini (2000, p. 1), avalando lo señalado anteriormente, concluye que "la publicación electrónica científica presenta una serie de ventajas en relación con la publicación impresa convencional, tanto para el editor científico como para el usuario final".

Teniendo en cuenta que las publicaciones electrónicas están asumiendo un papel relevante en el proceso de comunicación de los investigadores, y conscientes de la importancia de las publicaciones periódicas como medio de difusión de la producción del conocimiento científico, en este estudio se realizó un análisis de la publicación titulada Encontros Bibli: Revista Eletrônica de Biblioteconomia e Ciência da Informação. La investigación confirmó cuáles son las características de esta revista como canal de divulgación del conocimiento producido en el área de la ciencia de la información en Brasil, además de analizar el papel que viene desempeñando en la construcción del campo científico de la ciencia de la información en el país.

\section{Metodología}

Para caracterizar la publicación Encontros Bibli: Revista Eletrônica de Biblioteconomia e Ciência da Informação como canal de comunicación científica se tuvieron en cuenta tanto sus características extrínsecas y como las intrínsecas.

La investigación desarrollada tuvo carácter exploratorio y abarcó técnicas de investigación documental. Se utilizaron como objeto de estudio los números publicados entre 1996 y 2003. Para posibilitar el análisis propuesto se llevaron a cabo prácticas bibliométricas en general, y en especial el análisis de citación.

Liniers (1998) recuerda que fue Prichard quien definió la bibliometría en 1969, en un artículo del Journal of Documentation titulado "Statistical bibliography or bibliometrics". La bibliometría, para este autor, se define como la aplicación de métodos estadísticos y matemáticos a libros y otros medios de comunicación para clarificar los procesos de la comunicación escrita, así como la naturaleza y el desarrollo de las disciplinas científicas, utilizando técnicas de cómputo y análisis de las diferentes facetas de esta comunicación. El tratamiento y el manejo de la literatura científica a través del cómputo y del análisis sirven no solo para analizar el volumen de publicaciones y la productividad de autores, revistas o materiales, sino también, en un sentido más amplio, para el conocimiento de los procesos y la naturaleza de las ciencias. 
El estudio de citas es una rama de la bibliometría que investiga las relaciones entre los documentos citantes y los citados, en su totalidad o en parte (Braga, 1972). La cita bibliográfica sirve como indicador útil para el análisis del comportamiento de la literatura, es un instrumento fundamental en el proceso de recuperación de la información y tiene funciones reconocidas en el proceso de comunicación científica. Para Price (1974), la cita bibliográfica aporta el necesario reconocimiento de un científico por parte de sus colegas, indica la contribución científica de un autor (o publicación), ayuda a juzgar los hábitos de uso de la información y demuestra qué literatura es indispensable para el trabajo científico. Noronha (1998) afirma que el estudio de citas permite evaluar la información reunida en la literatura utilizada e indicar al lector otras fuentes de información sobre el tema, y además contribuye al reconocimiento del científico por sus colegas. La frecuencia de citas de un documento, para este autor, puede ser empleada como indicador de calidad. Cawel (1977) destaca diversas formas de utilizar los estudios: a) como medida del uso de la información, para establecer tasas de obsolescencia y ayudar a la selección de las publicaciones de una biblioteca; b) como medida del flujo de información en una comunidad científica; c) como indicador de calidad de la literatura; d) como análisis sociológicos de la ciencia; e) como indicadores de las tendencias científicas.

En esta investigación los términos periódico y revista se utilizaron con la misma acepción, y los materiales analizados se conceptualizaron de este modo: $a$ ) literatura fuente: la que constituye el material que ha servido de base para el estudio. En esta investigación, la literatura fuente fue la publicación Encontros Bibli: Revista Eletrônica de Biblioteconomia e Ciência da Informação; b) citación bibliográfica: conjunto de una o más referencias que, incluidas en una publicación, evidencian vínculos entre individuos, instituciones y áreas de investigación.

\section{Resultados}

En esta investigación se ha partido del presupuesto de que, ante el asombroso crecimiento de la literatura técnica científica, crear mecanismos que permitiesen medir esta producción equivalía a hacer posible la lectura del desarrollo de la ciencia en las diferentes áreas de estudio.

Esta cuestión gana aún más importancia cuando se aplica a la ciencia de la información, considerada como una ciencia emergente. Analizar la literatura publicada en revistas científicas en esta área permitiría delinear los aciertos y los desaciertos de las actividades de producción del conocimiento, posibilitando la creación de parámetros de evaluación y retroalimentación para esta producción.

Producción del conocimiento o producción científica, como comúnmente la denominan los autores, según Drew (ápud Gargantini, 1997, p. 59), es el proceso en el que interactúan el productor y el consumidor de la ciencia, interacción 
intermediada por un producto resultante de la acción del productor. Al estudiarla se puede hacer hincapié en el productor, en el consumidor, en el producto o en las relaciones procesales entre estos elementos. En esta investigación se ha incidido en las relaciones procesales existentes entre el producto (la publicación, los artículos y las citas) y los productores (los investigadores).

Se emplearon técnicas bibliométricas como herramientas válidas y eficaces para alcanzar los objetivos propuestos, o sea, para medir el flujo de información entre los autores de los artículos, caracterizar la producción y determinar la influencia de los autores, y como indicadores de tendencias teóricas.

\subsection{Características extrínsecas}

Para analizar las características extrínsecas se ha tenido en cuenta la estructura la publicación en sí, o sea, el consejo editorial, la periodicidad y el número de artículos por número.

Encontros Bibli: Revista Eletrônica de Biblioteconomia e Ciência da Informação fue la primera revista electrónica brasileña del área de la ciencia de la información disponible en Internet. Creada en 1996 por el Departamento de Ciencia de la Información de la Universidade Federal de Santa Catarina (UFSC), tiene una periodicidad semestral y está disponible en la URL http://www.encontrosbibli.ufsc.br. Hoy día se publica en coedición entre el Programa de Posgrado y el Departamento de Ciencia de la Información de la UFSC.

En la actualidad posee un comité editorial y un consejo editorial. La primera está formada por seis profesores del propio Departamento de Ciencia de la Información de la UFSC. El consejo se compone de seis investigadores; de ellos, cuatro tienen vínculos con instituciones nacionales (la Universidade Federal da Paraíba, la Universidade Federal do Ceará, la Universidade Estadual de São Paulo y la Universidade Estadual de Londrina) y dos con instituciones latinoamericanas (la Universidad de Buenos Aires y la Universidad de la República, de Montevideo). Los artículos que han de publicarse son enviados al editor, que los somete a la evaluación de tres revisores. El procedimiento de evolución se lleva a cabo mediante los métodos de peer-review (revisión por pares) y double-blind review (durante proceso de evaluación no se revela la identidad de los evaluadores ni la de los autores). Los evaluadores pueden formar parte del consejo editorial o del comité editorial, aunque también pueden ser revisores invitados ad hoc. Los artículos se distribuyen para su evaluación según los temas y su dominio por parte del revisor.

La revista Encontros Bibli admite artículos inéditos de biblioteconomía y ciencia de la información, de carácter teórico, basados en análisis de literatura, investigaciones y/o relatos de experiencias, según consta en las normas de publicación disponibles en su página web. En su plan editorial actual prevé la siguiente estructura para su organización interna: editorial, artículos y reseñas o libros recién 
editados. Al analizar los diferentes números de la revista se puede observar que la esta ha sufrido algunos cambios en su estructura. A lo largo de su existencia ha presentado secciones como comunicaciones de investigación, contribuciones de estudiantes, especiales, reseñas o publicaciones recibidas.

Creada en 1976 por iniciativa del profesor Francisco das Chagas Souza, del Departamento de Ciencia de la Información de la UFSC, la revista publicó en sus tres primeros números (1996-1997) tres artículos de este profesor, cuando aún no contaba con un comité editorial. A partir del número 4 (1997) se definió como una revista de investigación y opinión sobre educación biblioteconómica en Brasil y se constituyó un comité editorial que tenía como misión analizar las contribuciones intelectuales enfocadas al pensamiento y a la discusión pública sobre la educación biblioteconómica en Brasil. En este número apareció el primer artículo externo a la UFSC, de las profesoras Rosemberg y Correa (1997), que trataba de la creación y del reconocimiento del Curso de Biblioteconomía de la Universidade do Espirito Santo. El comité editorial estaba compuesto por profesores vinculados al Departamento de Ciencia de la Información de la UFSC y el editor era el profesor Francisco das Chagas de Souza. En el número 6 (1998) se observa una modificación en título de la publicación, que pasa caracterizarse como una revista de biblioteconomía y ciencia de la información. Este cambio se refleja igualmente en su contenido, que ahora tiene un enfoque más amplio, lo que posiblemente justifique el aumento significativo de la cantidad de artículos publicados por número. Este número da comienzo a una nueva fase de la revista y en él aparecen cinco artículos. Además se produce el ingreso de un componente externo a la UFSC en el comité editorial, del que pasa a formar parte la profesora Eliany Alvarenga de Araújo, de la Universidade Federal da Paraíba. En el número 8 (1999) tiene lugar un cambio en la coordinación del comité editorial, función que asume la profesora Miriam Vieira da Cunha, del Departamento de Ciencia de la Información de la UFSC, y la introducción de una sección denominada "Contribución de los estudiantes", con la intención de estimular a los alumnos de los cursos de Biblioteconomía para que publiquen artículos. A partir del número 9 (2000) la revista aparece registrada con el International Standard Serial Number (ISSN) 1518-2924. En el 10 (2000) se comunica una ampliación significativa del comité editorial, con el ingreso de varios evaluadores externos a la UFSC: Elsa Barber, de la Universidad de Buenos Aires; Mario Barité, de la Universidad de la República, de Montevideo; Marta Valentim, de la Universidade Estadual de Londrina, y José Augusto Guimarães, de la Universidade Estadual Paulista. En el número 14 (2002) la revista pasa a titularse Encontros Bibli: Revista Eletrônica de Biblioteconomia e Ciência da Informação y se constituye un comité editorial coordinado por el profesor Francisco das Chagas Souza, con capacidad ejecutiva, y un consejo editorial, con el objeto de reunir a los evaluadores externos a la UFSC que ya participaban en el 
proceso, incluyendo además a una nueva consejera, Maria de Fátima Portela Cysne, de la Universidade Federal do Ceará. En el número 15 (2003) se crea un nuevo proyecto gráfico y editorial para la revista. En el 16 (2003), la revista pasa a ser publicada en coedición entre el Departamento de Ciencia de la Información y el Programa de Posgrado en Ciencia de la Información, y es incluida en la Red de Revistas Científicas de América Latina y el Caribe, España y Portugal (REDALYC).

En el periodo de 1996 a 2003 se publicaron 16 números de la revista Encontros Bibli, según muestra la tabla I. Para el análisis que se ha llevado a cabo en esta investigación, se tuvieron en cuenta solamente los artículos, incluyendo los aparecidos como contribuciones de estudiantes y las comunicaciones de investigación, pero excluyendo los demás formatos publicados a lo largo de estos años.

\begin{tabular}{|c|c|c|}
\hline Año & Números & Artículos publicados \\
\hline 1996 & 1 & 1 \\
& 2 & 1 \\
\hline 1997 & 3 & 1 \\
& 4 & 1 \\
\hline 1998 & 5 & 1 \\
& 6 & 5 \\
\hline 1999 & 7 & 4 \\
& 8 & 5 \\
\hline 2000 & 9 & 3 \\
& 10 & 3 \\
\hline 2001 & 11 & 4 \\
& 12 & 5 \\
\hline 2002 & 13 & 3 \\
& 14 & 4 \\
\hline 2003 & 15 & 4 \\
& 16 & 4 \\
\hline & & 49 \\
\hline
\end{tabular}

Tabla I. Distribución de los artículos (fuente: Encontros Bibli, 1996-2003).

En el periodo analizado se publicó una media de 3 artículos por número. Este resultado es en parte consecuencia de la pequeña cantidad de artículos aparecidos en los primeros números de la revista (1-5), en los que se incluyeron 5 artículos, solamente uno en cada número. Esta baja cifra se debe probablemente al hecho de que esta fue la primera revista del área de biblioteconomía y ciencia de la información que se publicó en Internet, cuando la credibilidad del medio aún estaba en proceso de construcción y el desconocimiento por parte de los investigadores era consecuencia de que la revista estaba comenzando sus actividades. 
Sin embargo, hay que mencionar que, a pesar de la poca afluencia de artículos, la periodicidad semestral de la revista Encontros Bibli se mantuvo desde su aparición, lo que supone un indicador de calidad. Tanto Braga y Oberhofer (1982) como Müeller (1999) incluyen entre los factores de calidad de una revista científica el hecho de mantener la regularidad en su publicación. En Brasil, según Valério (1994), la periodicidad de las publicaciones científicas es un elemento muy importante y garantiza su credibilidad y su calidad, pues la mayoría no consigue cumplir sus plazos de publicación, probablemente debido a los elevados costos de producción y a la escasa inversión para ese fin en el país. La revista Encontros Bibli se publica en formato electrónico, y por eso su proceso de edición resulta más ágil y sencillo, y los costos derivados de su producción son menores.

\subsection{Características intrínsecas}

Para analizar las características intrínsecas se ha utilizado el tipo de autoría, la vinculación institucional y la productividad de los autores, las temáticas abordadas y las citas de los artículos que superaron la selección de los evaluadores de la revista, con la finalidad de comprobar las tendencias teóricas de los autores.

\section{Autoría y productividad de los autores}

En la revista Encontros Bibli se constata que, en el periodo de 1996 a 2003, se publicaron 33 artículos de autor único (el 67,4\% del total) y 16 escritos en colaboración $(32,6 \%)$, según muestra la tabla II. Este resultado confirma una tendencia del área de ciencias sociales ya verificada en estudios anteriores y en la propia ciencia de la información, según señalan Müller y Pecegueiro (2001).

\begin{tabular}{|c|c|c|c|}
\hline Tipo de autoría & Artículos & $\%$ & \\
\hline 1 autor & 33 & 67,4 & \\
\hline 2 autores & 8 & 16,3 & \\
\hline 3 autores & 4 & 8,2 & \\
\hline 4 autores & 2 & 4,1 & 32,6 \\
\hline 5 autores & 1 & 2 & \\
\hline 9 autores & 1 & 2 & \\
\hline Total & 49 & 100 & \\
\hline
\end{tabular}

Tabla II. Caracterización de la autoría (fuente: Encontros Bibli, 1996-2003).

Se registraron 61 autores, que escribieron los 49 artículos publicados en el periodo estudiado. De ellos se observa que 11 artículos fueron elaborados por un mismo autor, 5 por otro autor, 4 por 1 autor, 2 por 4 autores y 54 son obra de autores diferentes entre sí (tabla III). En este cálculo se atribuyó un artículo entero Scire. $12: 2$ (jul.-dic. 2006) 99-117. ISSN 1135-3716. 
a cada autor siempre que su nombre constara como tal, o sea, aunque el artículo hubiera sido escrito en colaboración. Por este motivo tuvo lugar un aumento artificial del número de artículos publicados, que pasó de 49 a 82. Los resultados indican que el autor más productivo fue responsable de 11 artículos; teniendo en cuenta la distorsión mencionada, le correspondería el 13,4\% de los 82 artículos contabilizados a este efecto y, en el otro extremo, 54 personas diferentes constaban solo una vez como autores de artículos publicados en la revista.

\begin{tabular}{|l|c|c|}
\hline Autores & & Artículos \\
\hline 1 autor & fue responsable de & 11 \\
\hline 1 autor & fue responsable de & 5 \\
\hline 1 autor & fue responsable de & 4 \\
\hline 4 autores & fueron responsables de & 2 \\
\hline 54 autores & fueron responsables de & 1 \\
\hline
\end{tabular}

Tabla III. Productividad de los autores (fuente: Encontros Bibli, 1996-2003).

Analizando la productividad de los autores, 3 de ellos destacan como los más productivos: Francisco das Chagas de Souza, con 11 artículos; Clarice Fortkamp Caldin, con 5, y Maria del Carmen Rivera Bohn, con 4. Se trata de profesores vinculados al Departamento de Ciencia de la Información de la UFSC y a la línea de investigación "Profesionales de la información" del Departamento y del Programa de Posgrado en Ciencia de la Información de la UFSC.

\section{Procedencia y vínculo institucional de los autores}

En la revista Encontros Bibli todos los artículos fueron escritos por autores que han estado vinculados a instituciones de enseñanza y/o investigación. La mayor parte de los autores se concentran en la categoría de docentes (profesores). En un estudio similar, Autran y Albuquerque (2002) observaron que en la revista brasileña del área de la ciencia de la información Informação \& Sociedade había una mayor participación de profesores y estudiantes como autores. Müeller y Pecegueiro (2001, p. 2), analizando la revista brasileña Ciência da Informação, también verificaron que en ella predominaban los profesores como autores, y afirmaron que este hecho no puede causar sorpresa, "pues investigar y escribir artículos forma parte de la carrera universitaria".

De los 61 autores computados, 38 (62,3\%) están vinculados a universidades, $28(73,6 \%)$ a universidades brasileñas y $10(26,4 \%)$ a universidades extranjeras $(9$ sudamericanas y 1 europea). En la categoría de estudiantes vinculados a universidades hay 18 autores $(29,5 \%)$. En la de técnicos de nivel superior encontramos $5(8,2 \%)$, vinculados a instituciones de investigación (tabla IV). 


\begin{tabular}{|l|c|c|c|c|}
\hline Institución & Vinculación & & & Total \\
\hline Universidade Federal de Santa Catarina, BR & 11 & 11 & 0 & 22 \\
\hline Universidade Federal de Minas Gerais, BR & 7 & 1 & 0 & 8 \\
\hline Universidad de Buenos Aires, AR & 8 & 0 & 0 & 8 \\
\hline Universidade do Estado de Santa Catarina, BR & 2 & 3 & 0 & 5 \\
\hline Fundação Oswaldo Cruz, BR & 0 & 0 & 3 & 3 \\
\hline Universidade Federal do Amazonas, BR & 0 & 2 & 0 & 2 \\
\hline Centro de Información FUSAT, AR & 0 & 0 & 1 & 1 \\
\hline City University, UK & 1 & 0 & 0 & 1 \\
\hline Instituto Politécnico Nacional, MX & 0 & 0 & 1 & 1 \\
\hline Universidade Estadual de Londrina, BR & 1 & 0 & 0 & 1 \\
\hline Universidade Federal do Espírito Santo, BR & 4 & 0 & 0 & 4 \\
\hline Universidade Federal Fluminense, BR & 1 & 0 & 0 & 1 \\
\hline Universidade Federal da Paraíba, BR & 1 & 0 & 0 & 1 \\
\hline Universidade Federal do Rio Grande do Sul, BR & 1 & 0 & 0 & 1 \\
\hline Universidade Estadual de São Paulo, BR & 0 & 1 & 0 & 1 \\
\hline Universidad de la República, UR & 1 & 0 & 0 & 1 \\
\hline Total & 38 & 18 & 5 & 61 \\
\hline
\end{tabular}

Tabla IV. Procedencia y vínculo institucional de los autores (fuente: Encontros Bibli, 1996-2003).

Se observa que, entre los autores, $22(36 \%)$ proceden de la Universidad Federal de Santa Catarina (UFSC); de ellos, 11 (50\%) son docentes y $11(50 \%)$ son estudiantes. Este hecho nos llama la atención por la tendencia endógena de la publicación. Según Valério (1994), esto debe ser evitado, pues dificulta el intercambio de ideas y puede llevar a la inercia y a la perpetuación de ideas, grupos y personas.

\section{Temáticas abordadas}

Todos los artículos publicados por la revista Encontros Bibli en el periodo analizado (1996-2003) están relacionados con el área de biblioteconomía y ciencia de la información (tabla V).

Los enfoques con mayor incidencia son los referidos a profesionales de la información (43\%), comunicación científica $(10,2 \%)$, lectura $(8,2 \%)$, organizaciones $(6,1 \%)$, preservación y conservación $(6,1 \%)$, bibliotecas académicas $(4,1 \%)$, cognición $(4,1 \%)$, sociedad de la información $(4,1 \%)$ y tecnologías de la información $(4,1 \%)$.

Se constata que los artículos relacionados con los profesionales de la información (21, es decir, el $43 \%$ de un total de 49 artículos), que abordaban temas

Scire. 12 : 2 (jul.-dic. 2006) 99-117. ISSN 1135-3716. 


\begin{tabular}{|l|c|c|}
\hline Temas & Artículos & $\%$ \\
\hline Profesionales de la información & 21 & 43 \\
\hline Comunicación científica & 5 & 10,2 \\
\hline Lectura & 4 & 8,2 \\
\hline Organizaciones & 3 & 6,1 \\
\hline Preservación y conservación & 3 & 6,1 \\
\hline Bibliotecas académicas & 2 & 4,1 \\
\hline Cognición & 2 & 4,1 \\
\hline Sociedad de la información & 2 & 4,1 \\
\hline Tecnologías de la información & 2 & 4,1 \\
\hline Bibliotecas públicas & 1 & 2 \\
\hline Extensión universitaria & 1 & 2 \\
\hline Indicadores & 1 & 2 \\
\hline Literatura infantil & 1 & 2 \\
\hline Movimiento estudantil & 1 & 2 \\
\hline Total & 49 & 100 \\
\hline
\end{tabular}

Tabla V. Temáticas abordadas (fuente: Encontros Bibli, 1996-2003).

referentes a la enseñanza, a la formación profesional, al perfil y a actuación de estos profesionales, fueron los más numerosos durante el periodo estudiado. Tal concentración temática se debe a varios factores: el propio origen de la revista Encontros Bibli, que tenía el propósito de estar destinada a la investigación y a la opinión sobre educación bibliotecaria en Brasil, y por eso en los cinco primeros números se publicaron artículos de esta temática; los temas están relacionados con líneas de investigación consolidadas en el Departamento de Ciencia de la Información de la UFSC, una de las cuales ("Profesionales de la información") forma parte del Programa de Posgrado en Ciencia de la Información. La distribución temática de los artículos en la revista también se vio afectada por la producción de los autores más prolíficos de la revista, puesto que el tema más repetido coincide con la línea de investigación de tales autores.

Análisis de las citas

Se contabilizaron 701 citas en los 49 artículos publicados, con un promedio de 14,30 citas por artículo (tabla VI).

A continuación sintetizamos los resultados más significativos obtenidos a través del estudio de citas.

1. Los materiales más utilizados como fuente de información fueron los libros con 287 citas (el $41 \%$ de un total de 701) y los artículos de periódicos con 


\begin{tabular}{|c|c|c|c|}
\hline Año & Número & Artículos publicados & Número de citas \\
\hline 1996 & 1 & 1 & 17 \\
& 2 & 1 & 13 \\
\hline 1997 & 3 & 1 & 4 \\
& 4 & 1 & 22 \\
\hline 1998 & 5 & 1 & 20 \\
& 6 & 5 & 51 \\
\hline 1999 & 7 & 4 & 27 \\
& 8 & 5 & 67 \\
\hline 2000 & 9 & 3 & 73 \\
& 10 & 3 & 41 \\
\hline 2001 & 11 & 4 & 75 \\
& 12 & 5 & 106 \\
\hline 2002 & 13 & 3 & 41 \\
& 14 & 4 & 35 \\
\hline 2003 & 15 & 4 & 54 \\
& 16 & 4 & 701 \\
\hline
\end{tabular}

Tabla VI. Distribución de las citas por artículos (fuente: Encontros Bibli, 1996-2003).

$216(31 \%)$. Entre los demás materiales citados, figuran documentos electrónicos con 48 citas $(6,8 \%)$ y trabajos publicados en eventos con 39 $(5,5 \%)$. Los artículos publicados en revistas electrónicas están encuadrados como artículos de periódicos, o sea, no se hizo distinción en cuanto al soporte. Los informes recibieron 23 citas (3,2\%); la legislación, 15 (2,1\%); las tesis de doctorado, solamente $3(0,4 \%)$, y las de maestría, $12(1,7 \%)$. En "otros" fueron encuadradas 58 referencias $(8,3 \%)$, relativas a entrevistas, testimonios, documentos administrativos (oficios, procesos y libros acta), anuarios, enciclopedias y almanaques.

2. Con relación al idioma, se percibe que el portugués fue el más citado, totalizando 542 citas (77,3\%). El inglés obtuvo 93 (13,3\%); el español, 37 $(5,3 \%)$; el francés, $26(3,7 \%)$, y $3(0,4 \%)$ recibió el alemán. En idioma portugués prevaleció el libro como el tipo de publicación más citada (44,3\%). Sin embargo, en inglés los artículos de revistas obtuvieron el mayor número de citas $(44,1 \%)$, lo mismo que en francés $(69,2 \%)$. En español hubo una aproximación entre el número de citas de libros $(37,8 \%)$ y el de artículos de revistas $(32,4 \%)$.

3. Por lo que respecta al área geográfica, Brasil fue el país que obtuvo más documentos citados: 531 fueron publicados en Brasil (75,7\%), 45 en Estados Unidos (6,4\%), 29 en Francia (4,1\%), 16 en Inglaterra (2,3\%) y 13 
en España (1,9\%). En Brasil, el 76,4\% de los documentos citados, que recibieron más de 20 citas, fueron publicados en las ciudades de São Paulo $(24,8 \%)$, Florianópolis (13\%), Brasilia (13\%), Río de Janeiro (10\%), Campinas $(7,7 \%)$, Porto Alegre (4\%) y Belo Horizonte (3,9\%). Esas ciudades, excepto Brasilia, están ubicadas en las regiones sur y sudeste del país, donde, según Targino y Garcia (2000, p. 107), se concentra el mayor número de investigadores, cursos posgrado, grandes editoriales y empresas, así como los más poderosos medios de comunicación. Autran y Albuquerque $(2002$, p. 2) resaltan que "en las regiones sur/sudeste se encuentran las mejores y más tradicionales universidades, los centros de investigación con reconocimiento internacional y los más importantes polos industriales". Aunque no esté ubicada en estas regiones, a Brasilia se le pueden atribuir las mismas condiciones por ser la capital del país. Es importante, además, señalar que la gran incidencia de documentos citados en esta investigación publicados en estas capitales se debe también a la ubicación de las universidades que desarrollan actividades relacionadas al área de la ciencia de la información y la biblioteconomía.

4. En cuanto al análisis de la edad de los documentos citados, se tomaron los siguientes intervalos temporales para encuadrar las referencias: hasta 2 años, de 3 a 4 años, de 5 a 6 años, de 7 a 10 años, de 11 a 20 años, más de 20 años y sin fecha. Se comprobó que 169 citas correspondían a documentos encuadrados en el intervalo de 7 a 10 años (24\%); 132, en el de 11 a 20 años $(18,9 \%)$, y 129, en el de 5 a 6 años $(18,4 \%)$. De las publicaciones de entre 7 y 10 años, $87(49,1)$ son libros y $57(33,7 \%)$ son artículos de periódicos. En las de entre 11 y 20 años, el libro también prevalece como el más citado (56,1\%), seguido por los artículos de periódicos (31,8\%). Los artículos de periódicos citados eran los más recientes; de estos, el 28,3\% se encuadraban en el intervalo de hasta 2 años, el 33,9\% tenían entre 3 y 4 años, y $38,8 \%$, entre 5 y 6 .

5. Al verificar el grupo de autores brasileños más citados, se observa que destacan Francisco das Chagas de Souza con 39 citas, Suzana Pinheiro Machado Müeller con 9, Mara Eliane Fonseca Rodrigues con 9, Aldo de Albuquerque Barreto con 6 y Eduardo Wense Dias con 6. Los autores extranjeros más citados fueron Michel J. Menou con 9 citas, Peter L. Berger y Thomas Luckmann con 7, Pierre Lévy con 6 y Manuel Castells con 5.

6. En el análisis de las citas a autores nacionales se detectó el fenómeno de la autocita: se observa que 61 (8,7\%) de ellas fueron autocitas. Según Freitas (1997, p. 126), estas, "aunque sean consideradas por algunos autores un tipo normal de cita, no representan el impacto de un trabajo (o autor) sobre otro"; por eso, en esta investigación, en este punto se optó por presentar una 
nueva evaluación de los autores citados. Una vez excluidas las autocitas, el grupo de autores más citados cambió, de modo que destacaban como autores nacionales más citados Susana Pinheiro Machado Müeller con 9 citas, Aldo de Albuquerque Barreto con 6, Eduardo Wense Dias con 6, José Augusto Chaves Guimarães con 5, Maria Lourdes Blatt Ohira con 5 y Ezequiel Teodoro da Silva con 5. Los autores extranjeros más citados fueron Peter L. Berger y Thomas Luckmann con 7 citas, Pierre Lévy con 6 y Mauel Castells con 5 .

7. Por lo que se refiere la literatura que es objeto del área, se constata que las revistas nacionales del área de la ciencia de la información lideran la lista de las más citadas: Ciência da Informação (25 citas), Transinformação (19), Informação \& Sociedade: Estudos (12) y Revista da Escola de Biblioteconomia da UFMG (11) (en 1997 esta publicación pasó a denominarse Perspectivas em Ciência da Informação, y con el nuevo título recibió 5 citas), Revista de Biblioteconomia de Brasília (9), Encontros Bibli (8) y DataGramaZero (7). Entre las revistas extranjeras que recibieron citas se destacan Bulletin des Bibliothèques de France (6 citas), Public Libraries (5), Annual Review of Information Science and Technology (4) y Documentaliste: Sciences de l'Information (4).

Respecto a los resultados obtenidos en el estudio de citas es importante comentar algunas cuestiones:

1. Los tipos de materiales más citados corresponden a los más tradicionales del proceso de comunicación científica: libros y revistas científicas. Se obtuvieon resultados similares en los estudios realizados para la revista Ciência da Informação por Müller y Pecegueiro (2001) y para Informação \& Sociedade por Autran y Albuquerque (2002).

2. El hecho de que el idioma portugués esté más presente en las citas se debe a los temas tratados, que casi siempre se refieren a la realidad del Brasil y, consecuentemente, requieren soporte teórico de autores brasileños. Velho (1997, p. 4) afirma que existen diferencias entre las ciencias en lo que se refiere al idioma y al ámbito geográfico de publicación, y que "la tendencia observada en los científicos de las áreas de humanas y sociales a publicar con más frecuencia en su país y en su propia lengua se debe, en parte, al propio objeto de estudio de estas áreas".

3. Los autores más citados son profesores/docentes de universidad y/o investigadores de institutos vinculados a la red pública de enseñaza e investigación de Brasil. En este país ya existe un consenso sobre que las instituciones públicas son las que desarrollan la investigación y, en consecuencia, son responsables de gran parte de la producción científica

Scire. 12 : 2 (jul.-dic. 2006) 99-117. ISSN 1135-3716. 
publicada en las revistas de todas las áreas del conocimiento, lo que se confirma para el área de la ciencia de la información en este estudio sobre la revista Encontros Bibli.

4. Las revistas nacionales más utilizadas como fuente de información son publicadas por instituciones públicas de enseñanza superior, con excepción de Transinformação, que es editada por una institución de enseñanza privada, la Pontifícia Universidade Católica de Campinas (PUCCAMP); Ciência da Informação, que lo es por el Instituto Brasileiro de Informação Científica e Tecnológica (IBICT), y DataGramaZero, que lo es por una organización no gubernamental, el Instituto de Adaptação e Inserção na Sociedade da Informação (IASI). Son revistas consolidadas y tienen un papel importante en el escenario de la divulgación de la producción del conocimiento en el área de la ciencia de la información en Brasil.

\section{Conclusión}

Esta investigación ha tenido por objeto contribuir a una mejor comprensión de la producción del conocimiento en el área de la ciencia de la información y la biblioteconomía en Brasil a través del análisis de la parte de esta producción que fue difundida a través de la publicación Encontros Bibli: Revista Eletrônica de Biblioteconomia e Ciência da Informação.

El estudio ha limitado su área de interés a la propia revista: estructura, autores, artículos publicados, evolución y desarrollo como medio de divulgación de la producción del conocimiento. Se ha partido del presupuesto de que la producción científica se refleja en las publicaciones periódicas editadas y que al estudiar artículos aparecidos en ellas en realidad se está analizando la literatura de una ciencia, o sea, su producto formalizado. De esta forma, la revista científica refleja la ciencia producida, y a través de ella quizá se pueda valorar el nivel de desarrollo de esa ciencia aunque se desconozca la naturaleza de ese reflejo (Pinheiro, 1983).

En su trayectoria como medio de comunicación científica se percibe que la revista Encontros Bibli empezó su proceso de estabilización a partir del año 1998 (número 6), con la ampliación de su contenido temático. A partir de ese momento ha habido algunos hitos importantes en su proceso evolutivo: 1999 (número 7), con la obtención del registro en el ISSN; 2000 (número 10), con la inclusión de miembros de la comunidad latinoamericana biblioteconómica en el comité editorial; 2003 (número 16), con su inclusión en un indexador de gran alcance como el REDALYC. Hay que resaltar que en el periodo analizado también mantuvo la periodicidad en su publicación, lo que supone un indicador seguro de calidad.

Aunque haya ampliado su contenido temático a partir de 1998, la revista Encontros Bibli ha contribuido de un modo más efectivo a la construcción del campo 
de la ciencia de la información y de la biblioteconomía en Brasil a través de la publicación de estudios que tratan sobre cuestiones relacionadas con los profesionales de la información, entre las que se incluyen las relativas a la educación, la formación, la actividad y el perfil de estos profesionales.

Tanto los autores que publicaron en la revista Encontros Bibli como los autores brasileños citados por ellos tienen vinculación con instituciones públicas brasileñas de enseñanza e investigación, lo que confirma que tales instituciones, también en el área de la ciencia de la información, son responsables de la producción del conocimiento científico en el país.

Los autores que más han influido en el desarrollo de los puntos de vista expuestos en la revista han sido Susana Pinheiro Machado Müeller, Aldo de Albuquerque Barreto, Eduardo Wense Dias, José Augusto Chaves Guimarães, Maria Lourdes Blatt Ohira y Ezequiel Teodoro da Silva. De ellos, un gran número (el $67 \%$ ) están vinculados a programas de posgrado del área de la ciencia de la información en Brasil, lo que confirma el papel relevante de estos programas en la construcción del conocimiento científico del área. Las regiones sur y sudeste y la ciudad de Brasilia se han identificado como focos productores de la información utilizada por los autores que publicaron en la revista, lo que coincide con la localización de los programas de posgrado existentes en el país. Los autores extranjeros más influyentes han resultado ser Peter L. Berger, Thomas Luckmann, Pierre Lévy y Manuel Castells, los cuales poseen un mayor grado de aceptación entre los autores que han publicado en Encontros Bibli. Para Volpato (2001) el fundamento teórico de las investigaciones se hace basándose en textos que los investigadores conocen y aceptan. No es suficiente publicar: es necesario que el texto sea localizado, leído y aceptado. La aceptación (cita) se ha convertido hoy día en un parámetro de calidad de la producción científica.

El análisis de lo que se está publicando en periódicos especializados ha demostrado ser uno de los caminos para sistematizar informaciones que complementen los esfuerzos por ampliar la comprensión de la producción del conocimiento en ciencia de la información y biblioteconomía en Brasil. Estudios de esta naturaleza son esenciales para identificar y trazar el mapa de los autores, conocer su productividad, las temáticas de mayor incidencia, las relaciones, las tendencias y los influjos más presentes en los documentos publicados.

La investigación ha sido importante para la comprensión de la producción del conocimiento porque "la literatura periódica se caracteriza por ser representativa de la comunidad productora en la medida en que su política editorial funciona como un filtro de calidad en el proceso de selección de artículos, siendo fragmentaria en la medida en que se construye por etapas a partir de trabajos anteriores y derivativa en la medida en que se constituye en apoyo para trabajos posteriores" (Foresti, 1990, pp. 53-54). La publicación de la producción del conocimiento promueve un

Scire. 12 : 2 (jul.-dic. 2006) 99-117. ISSN 1135-3716. 
flujo de interacción e integración de ideas en la comunidad productora, lo que se puede percibir en este estudio sobre Encontros bibli: Revista Eletrônica de Biblioteconomia e Ciência da Informação.

\section{Referencias}

Associação Brasileira de Normas Técnicas (2003). NBR 6021. Informação e Documentação: publicação periódica científica impressa. Apresentação. Río de Janeiro: ABNT, 2003.

Autran, M. de M. M.; Albuquerque, M. E. B. C. De (2002). Mapeamento do periódico Informação \& Sociedade: Estudos, dez anos de sua trajetória. // Informação \& Sociedade: Estudos, 12:1 (jan.-jun. 2002) 1-22. http://www.informacaoesociedade.ufpb.vbr (2003-04-12).

Braga, G. M. (1972). Relações bibliométricas entre a frente de pesquisa (research front) e revisões da literatura: estudo aplicado à ciência da Informação. Dissertação (mestrado em Ciência da Informação). Río de Janeiro: IBICT: Universidade Federal do Rio de Janeiro, 1972.

Braga, G. M.; Oberhofer, C. A. (1982). Diretrizes para a avaliação de periódicos científicos e técnicos brasileiros. // Revista Latinoamericana de Documentación. 2:1 (1982) 27-31.

Cawel, A. E. (1977). Citation as sociological and scientific indicators: a review. Ámsterdan: ASLIB, 1977. 31-39.

Dias, G. A. (2002). Periódicos eletrônicos: consideração à aceitação deste recurso pelos usuários. // Ciência da Informação. 31:3 (set.-dez. 2002) 18-25.

Foresti, N. A. B. (1990). Contribuição das revistas brasileiras de biblioteconomia e ciência da informação enquanto fonte de referência para a pesquisa. // Ciência da Informação. 19:1 (jan.-jun. 1990) 53-71.

Freitas, M. H. de A. (1997). Oito anos de Transinformação. // Transinformação. 9:3 (set.dez. 1997) 120-134.

Garcia, J. C. R.; Targino, M. das G. (1999). Restruturação de Informação \& Sociedade: Estudos, periódico do Curso de Mestrado em Ciência da Informação da Universidade Federal da Paraíba. // Informação \& Sociedade: Estudos. 9:1 (1999) 1-40.

Gargantini, M. B. M. (1997). Autoria de artigos do Journal of Fluency Disorders. // Transinformação. 9:3 (set.-dez. 1997) 53-67.

Greene, L. J. (1998). O dilema de uma revista biomédica: aceitar ou não aceitar. // Ciência da Informação. 27:2 (mai.-ago. 1998) 203-233.

Herscham, A. (1970). The primary journal: past, present and future. // Journal of Chemical Documentation. 10:1 (1970) 37-40.

Liniers, M. C. R. (1998). Bibliometría y ciencias sociales. // Clío. 7 (1998). http://clio. rediris.es/numero007.html (2003-03-23).

Meadows, A. J. (1999). A comunicação científica. Brasilia: Briquet de Lemos, 1999.

Moutinho, K.; Cunha Filho, P. C.; Lima, A. M. de (2002). Liber: alternativa para publicação eletrônica. // Ciência da Informação. 31:2 (mai.-ago. 2002) 80-85.

Scire. 12 : 2 (jul.-dic. 2006) 99-117. ISSN 1135-3716. 
Müeller, S. P. M. (1999). O círculo vicioso que prende os periódicos nacionais. // DataGramaZero. 0 (dez. 1999). http://www.dgzero.org/dez99/Art_04.htm (2003-03-31).

Müeller, S. P. M.; Pecegueiro, C. M. P. de (2001). O periódico Ciência da Informação na década de 90: um retrato da área refletido em seus artigos. // Ciência da Informação. 30:2 (mai.-ago. 2001) 47-63.

Noronha, D. P. (1998). Análise das citações das dissertações de mestrado e teses de doutorado em saúde pública (1990-1994): estudo exploratório. // Ciência da Informação. 27:1 (jan.-abr. 1998) 66-75.

Oliveira, E. da C. P. (1996). Revistas eletrônicas: papel ou bytes. // Informare: Caderno do Programa de Pós-graduação em Ciência da Informação. 2:1 (jan.-jun. 1996) 81-87.

Pinheiro, L. V. (1983). Lei de Bradford: uma reformulação conceitual. // Ciência da Informação. 12:2 (jul.-dez. 1983) 60-69.

Price, D. J. S. (1974). Society's need in scientific and technical information. // Ciência da Informação. 3:2 (1974) 97-103.

Rosemberg, D. S.; Corrêa, L. H. M. C. (1997). A criação e o reconhecimento do Curso de Biblioteconomia da UFES. // Encontros Bibli. 4 (set. 1997) 1-12. http://www.encontrosbibli.ufsc.br (2003-12-13).

Sabbatini, M. (1999). As publicações eletrônicas dentro da comunicação científica. // Encontro Lusófono das Ciências da Comunicação. 3 (1999). http://bocc.ubi.pt/pag/ sabbatini-marcelo-publicações-eletrocnicas.html (2003-04-01).

Sabbatini, M. (). Qualidade da informação nas publicações científicas electrônicas na Internet: desafios e propostas. // Teoría de la Educación: Educación y Cultura en la Sociedad de la Información. 2 (nov. 2000). http://www3.usal.es/teoriaeducacion/rev_ numero_02/n2_art_sabbatini.htm (2002-05-01).

Targino, M. das G. (2000). Comunicação científica: uma revisão de seus elementos básicos. // Informação e Sociedade: Estudos. 10:2 (2000) 37-85.

Targino, M. das G; Garcia, J. C. R. (2000). Ciência brasileira na base de dados do Institute for Scientific Information (ISI). // Ciência da Informação. 29 (jan.-abr. 2000) 103-117.

Valério, P. M. (1994). Espelho da ciência: avaliação do Programa Setorial de Publicações em Ciência e Tecnologia da FINEP. Río de Janeiro: FINEP; Brasilia: IBICT, 1994.

Velho, L. (1997). A ciência e seu público. // Transinformação. 9:3 (set.-dez. 1997) 15-32.

Volpato, G. L. (2001). Ciência: da filosofia à publicação. Jaboticabal: FUNEP, 2001. 3. a ed.

Ziman, J. M. (1979). Comunidade e comunicação. // Conhecimento público. São Paulo: Itatiaia: EDUSP, 1979. 115-130.

Scire. 12 : 2 (jul.-dic. 2006) 99-117. ISSN 1135-3716. 